\title{
(6) OPEN ACCESS \\ Triheptanoin dramatically reduces paroxysmal motor disorder in patients with GLUT1 deficiency
}

\author{
Fanny Mochel, ${ }^{1,2}$ Elodie Hainque, ${ }^{1,3}$ Domitille Gras, ${ }^{1,4}$ Isaac M Adanyeguh, ${ }^{1}$ \\ Samantha Caillet, ${ }^{5}$ Bénédicte Héron, ${ }^{6}$ Agathe Roubertie, ${ }^{7,8}$ Elsa Kaphan, ${ }^{9}$ \\ Romain Valabregue, ${ }^{1,10}$ Daisy Rinaldi, ${ }^{1}$ Sandrine Vuillaumier, ${ }^{11}$ Raphael Schiffmann, ${ }^{12}$ \\ Chris Ottolenghi, ${ }^{13,14}$ Jean-Yves Hogrel, ${ }^{15}$ Laurent Servais, ${ }^{16}$ Emmanuel Roze ${ }^{1,3}$
}

\begin{abstract}
- Additional material is published online only. To view please visit the journal online (http://dx.doi.org/10.1136/ jnnp-2015-311475).

For numbered affiliations see end of article.

\section{Correspondence to} Dr Fanny Mochel, Institut du Cerveau et de la Moelle épinière, Aile 4A, Groupe Hospitalier Pitié-Salpétrière, Paris 75013, France; fanny.mochel@upmc.fr
\end{abstract}

Received 2 July 2015 Revised 29 September 2015 Accepted 14 October 2015 Published Online First 4 November 2015

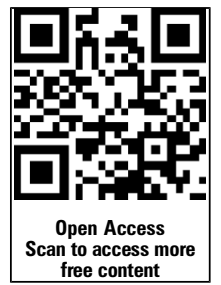

CrossMark

To cite: Mochel $F$ Hainque $\mathrm{E}$, Gras $\mathrm{D}$, et al. $J$ Neurol Neurosurg Psychiatry 2016;87: 550-553.

\section{ABSTRACT}

Objective On the basis of our previous work with triheptanoin, which provides key substrates to the Krebs cycle in the brain, we wished to assess its therapeutic effect in patients with glucose transporter type 1 deficiency syndrome (GLUT1-DS) who objected to or did not tolerate ketogenic diets.

Methods We performed an open-label pilot study with three phases of 2 months each (baseline, treatment and withdrawal) in eight patients with GLUT1-DS (7-47 years old) with non-epileptic paroxysmal manifestations. We used a comprehensive patient diary to record motor and non-motor paroxysmal events. Functional ${ }^{31} \mathrm{P}$-NMR spectroscopy was performed to quantify phosphocreatine ( $\mathrm{PCr}$ ) and inorganic phosphate (Pi) within the occipital cortex during (activation) and after (recovery) a visual stimulus.

Results Patients with GLUT1-DS experienced a mean of $30.8( \pm 27.7)$ paroxysmal manifestations (52\% motor events) at baseline that dropped to $2.8( \pm 2.9,76 \%$ motor events) during the treatment phase $(p=0.028)$. After withdrawal, paroxysmal manifestations recurred with a mean of $24.2( \pm 21.9,52 \%$ motor events; $\mathrm{p}=0.043)$. Furthermore, brain energy metabolism normalised with triheptanoin, that is, increased $\mathrm{Pi} / \mathrm{PCr}$ ratio during brain activation compared to the recovery phase $(p=0.021)$, and deteriorated when triheptanoin was withdrawn.

Conclusions Treatment with triheptanoin resulted in a 90\% clinical improvement in non-epileptic paroxysmal manifestations and a normalised brain bioenergetics profile in patients with GLUT1-DS.

Trial registration number NCT02014883.

\section{INTRODUCTION}

Glucose transporter type 1 deficiency syndrome (GLUT1-DS) is caused by impaired glucose transport across the blood-brain barrier and into astrocytes, leading to cerebral energy deficiency. ${ }^{1}$ GLUT1-DS is caused by mutation in the SLC2A1 gene encoding the glucose transporter GLUT1. The phenotype typically comprises psychomotor retardation and permanent motor disorders, associated with paroxysmal manifestations including seizures and non-epileptic paroxysmal episodes. ${ }^{1} 2$ With age, seizures tend to become less prominent, whereas the frequency of non-epileptic paroxysmal episodes increases. ${ }^{3}$ In patients with milder forms of the disease, paroxysmal movement disorders, especially dyskinesia, may be the main or the sole manifestations of the disease and can occur at any age. $^{3} 4$ Ketogenic diets, which provide ketone bodies to the brain and compensate for the lack of glucose, represent the standard of care in GLUT1-DS ${ }^{15}$ and are efficient on seizures control but less on movement disorders. ${ }^{2}$ Moreover, many patients, especially adolescents and adults, have difficulties in complying with the difficult constraints of these long-term diets and their side effects.

Triheptanoin is an odd-chain triglyceride with anaplerotic properties-that is, replenishing the pool of metabolic intermediates in the Krebs cycle. Unlike even-chain fatty acids metabolised to acetyl-CoA only, triheptanoin can indeed provide both acetyl-CoA and propionyl-CoA, two key carbon sources for the Krebs cycle. We showed that triheptanoin was able to produce C5-ketone bodies and restore energy metabolism and neurotransmission in pyruvate carboxylase (PC) deficiency, a severe metabolic disease that affects anaplerosis in the brain. ${ }^{6}$ Recently, we demonstrated that triheptanoin is able to correct bioenergetics in the brain of patients with Huntington's disease (HD), a neurodegenerative disease associated with brain energy deficit. ${ }^{7}$ Here, we wished to obtain a proof-of-concept of the therapeutic effect of triheptanoin in patients with GLUT1-DS with non-epileptic paroxysmal manifestations for whom a ketogenic diet was not a therapeutic option.

\section{SUBJECTS AND METHODS}

Participants were enrolled in an interventional clinical protocol (NCT02014883) at the Pitié-Salpêtrière Hospital promoted by INSERM and approved by the local ethical committee. All participants and/or their legal guardians signed a written informed consent before participating in the study. Four children and four adults were enrolled with GLUT1-DS as defined by low cerebrospinal fluid to blood glucose ratio and a SLC2A1 missense mutation predicted to be pathogenic by prediction software programs. Patients had a chronic history of non-epileptic paroxysmal motor disorders, especially paroxysmal exercise-induced dyskinesia, nonkinesigenic dyskinesia, limb weakness, headache, drowsiness and dysphoria. Three patients also presented a mild cognitive deficit. All patients were on a normal diet prior to their enrolment as either they objected to or did not tolerate ketogenic diets. The 
study was divided into three phases of 2 months each (baseline, treatment and withdrawal). A trained dietician determined the patient's caloric intake and adjusted their daily menus so that their diet remained isocaloric when triheptanoin was introduced. During the treatment phase, each patient was required to ingest $1 \mathrm{~g} / \mathrm{kg}$ body-weight of triheptanoin per day, divided into 3-4 intakes during meals.

During each study phase, the patients and/or their legal guardians had to fill a comprehensive patient diary to record all motor (seizure, abnormal movement, body stiffness, body weakness, abnormal speech) and non-motor (headache, lethargy, mood swing) paroxysmal events (see online supplementary table e1). The approximate duration of each episode was recorded
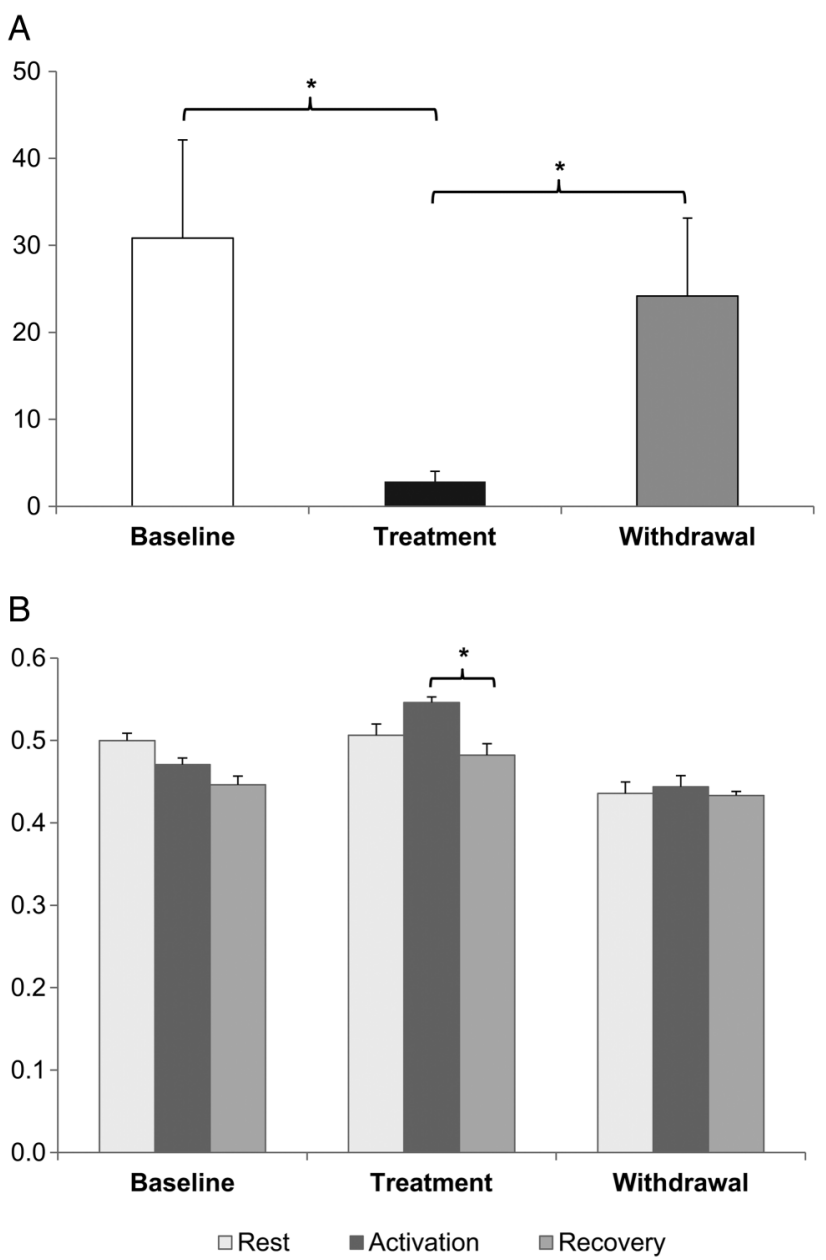

Figure 1 (A) Number of total paroxysmal manifestations in patients with glucose transporter type 1 deficiency syndrome (GLUT1-DS) during the three phases of the study (baseline, treatment and withdrawal) of 2 months each. A significant reduction of non-epileptic paroxysmal manifestations was observed when patients were treated with triheptanoin for 2 months $\left({ }^{*} p<0.05\right)$. Of note, the total number of events was comparable between the baseline and withdrawal phases. Error bars represent SEM. (B) Changes in the inorganic phosphate and phosphocreatine ( $\mathrm{Pi} / \mathrm{PCr}$ ) ratio from ${ }^{31} \mathrm{P}-\mathrm{NMR}$ spectroscopy (f-MRS) studies during the three phases of the study (baseline, treatment and withdrawal). During baseline, f-MRS showed an abnormal brain energy profile in patients with GLUT1-DS with no change in the Pi/PCr ratio during visual stimulation. After 2 months of treatment with triheptanoin, the profile was corrected and we observed an increase in the $\mathrm{Pi} / \mathrm{PCr}$ ratio during visual stimulation followed by a decrease during recovery ( $\left.{ }^{*} p=0.021\right)$. Error bars represent SEM of within-subject differences using the method of Morey. (minutes). At each visit, recordings of all paroxysmal events were reviewed with the evaluating physician and items were grouped into motor and non-motor episodes. At each visit, patients were also evaluated with a 6 min walk test (6MWT), a nine-hole pegboard test (9HPT) and the clinical global impression-improvement scale (CGI-I). The 6MWT was performed in a corridor, between two cones separated by a distance of $25 \mathrm{~m}$, in order to get the maximal distance covered during $6 \mathrm{~min}$ by walking. Both the dominant and non-dominant hands were tested twice during the 9HPT and the best score for each hand was recorded. Blood samples were collected after an overnight fast for standard analyses, plasma C3-carnitine and C5-ketone bodies. ${ }^{6}$

To assess the effect of triheptanoin on brain energy metabolism, functional ${ }^{31} \mathrm{P}-\mathrm{NMR}$ spectroscopy (f-MRS) was performed at $3 \mathrm{~T}$ at the end of each study phase in patients $>15$ years old $(n=5)$. We targeted the visual cortex using a surface coil as it is easily stimulated and is close to the scalp, allowing an increased sensitivity to the surface coil. Furthermore, visual stimulation results in a large increase in glucose uptake and cerebral blood flow. ${ }^{8}$ Data were collected for $4 \mathrm{~min}$ at rest, 8 min during visual activation, $8 \mathrm{~min}$ after stimulation and analysed as described. ${ }^{7} 9$ The ratio of inorganic phosphate over phosphocreatine (Pi/PCr) was calculated to determine the brain response to cortical activation as it is directly related to the ADP levels which regulate mitochondrial oxidative metabolism. ${ }^{10}$

Paired $t$ tests were used for plasma analyses before and after treatment. For clinical parameters, Friedman tests were used to test the global hypothesis that all study phases were equal. If significant, Wilcoxon signed-rank tests were applied for pairwise phase comparisons with an $\alpha$ of 0.05 . For the $\mathrm{Pi} / \mathrm{PCr}$ ratio, repeated measures analysis of variance (ANOVA) were used to test the global hypothesis that all time points (rest, activation and recovery) were equal. If significant, paired $t$ tests were applied for pairwise time comparisons with an $\alpha$ of 0.05 .

Table 1 Main characteristics of patients with GLUT1-DS during the baseline, treatment and withdrawal phases

\begin{tabular}{|c|c|c|c|c|c|c|c|}
\hline & \multicolumn{6}{|c|}{ Patients } & \multirow[b]{2}{*}{ Mean } \\
\hline & P1 & P2 & P3 & P4 & P5 & P6 & \\
\hline Sex & $\mathrm{F}$ & $\mathrm{F}$ & M & M & M & M & \\
\hline Age & 23 & 20 & 7 & 14 & 16 & 47 & 21.2 \\
\hline \multicolumn{8}{|l|}{ Baseline } \\
\hline Total events & 10 & 20 & 13 & 26 & 31 & 85 & 30.8 \\
\hline Motor events & 6 & 16 & 10 & 1 & 12 & 54 & 16.5 \\
\hline 6MWT (m) & 502 & 558 & 458 & 514 & 471 & 504 & 501 \\
\hline 9HPT(D/ND) (s) & $17 / 18$ & $18 / 23$ & $19 / 21$ & $15 / 17$ & $18 / 22$ & $14 / 16$ & $17 / 19$ \\
\hline \multicolumn{8}{|l|}{ Treatment } \\
\hline Total events & 4 & 7 & 5 & 0 & 1 & 0 & 2.8 \\
\hline Motor events & 2 & 7 & 3 & 0 & 1 & 0 & 2.2 \\
\hline 6MWT (m) & 453 & 580 & 425 & 484 & 496 & 500 & 490 \\
\hline 9HPT(D/ND) (s) & $17 / 19$ & $17 / 20$ & $19 / 19$ & $17 / 21$ & $17 / 18$ & $15 / 18$ & $17 / 19$ \\
\hline \multicolumn{8}{|l|}{ Withdrawal } \\
\hline Total events & 10 & 11 & 5 & 20 & 36 & 63 & 24.2 \\
\hline Motor events & 9 & 9 & 1 & 1 & 15 & 40 & 12.5 \\
\hline 6MWT (m) & 532 & 528 & 461 & 496 & 475 & 510 & 500 \\
\hline 9HPT(D/ND) (s) & $15 / 22$ & $14 / 20$ & $17 / 17$ & $17 / 19$ & $18 / 20$ & $15 / 15$ & $16 / 19$ \\
\hline
\end{tabular}

Total events: all motor and non-motor paroxysmal manifestations during each 2-month-phase. Motor events: all motor paroxysmal episodes during each 2-month-phase. 6MWT: Total distance walked (metres). 9HPT-D: the best score (seconds) obtained with the dominant hand. 9HPT-ND: best score (seconds) with the non-dominant hand.

GLUT1-DS, glucose transporter type 1 deficiency syndrome; 6MWT, 6 min walk test; 9HPT, nine-hole pegboard test 


\section{RESULTS}

Triheptanoin was well tolerated in all patients and none experienced gastrointestinal symptoms. Two patients were considered not compliant with the study as they consumed less than $50 \%$ of the recommended dose of triheptanoin and they (or their legal guardians) regularly omitted to fill the patient diary: a 13-year-old patient with mild cognitive delay who experienced only a few paroxysmal manifestations prior to the study, and a 23-year-old patient who was in denial of his disease. Owing to the small sample size, a per-protocol analysis was performed in the six patients who completed the study (see online supplementary table e2).

During the baseline phase, patients with GLUT1-DS experienced a mean of $30.8( \pm 27.7,10-85)$ paroxysmal manifestations over 2 months, including 16.5 ( $\pm 19.1,1-54)$ motor episodes. When treated with triheptanoin for 2 months, paroxysmal manifestations dropped to a mean of 2.8 ( $\pm 2.9,0-7)$, including $2.2( \pm 2.6,0-7)$ motor episodes $(\mathrm{p}=0.028$, figure $1 \mathrm{~A})$, representing overall a $90 \%$ symptoms reduction. Two patients became free from paroxysmal manifestations (table 1). Although not significant, triheptanoin tended to also reduce the mean duration of the remaining motor episodes $-59 \pm 44 \mathrm{~min}$ at baseline compared to $10 \pm 10 \mathrm{~min}$ with triheptanoin, $p=0.224$. On the patient-rated CGI-I scale, all patients reported a clear improvement when treated ('much improved'). Conversely, during the 2-month withdrawal phase, patients experienced a mean of $24.2( \pm 21.9,5-63)$ paroxysmal manifestations, including $12.5( \pm 14.5,1-40)$ motor episodes $(p=0.043$, figure $1 \mathrm{~A})$. On the patient-rated CGI-I scale, five out of six patients reported a clear worsening during withdrawal ('much worse'). The patients' performance on the 6WMT and 9HPT was unchanged along the different phases of the study (table 1). On study completion, all patients wished to continue treatment with triheptanoin.

Compared to baseline, we observed a significant increase in plasma C3-carnitine $(\mathrm{p}=0.026)$ and C5-ketone bodies $(p=0.008)$ on triheptanoin, reflecting its proper metabolism in the six compliant patients with GLUT1-DS. Conversely, the levels of triheptanoin metabolites were unchanged in the two non-compliant patients. During baseline, f-MRS showed an abnormal profile with no change in the $\mathrm{Pi} / \mathrm{PCr}$ ratio during brain activation in patients with GLUT1-DS (figure 1B), unlike what we reported in healthy individuals. ${ }^{79}$ After 2 months on triheptanoin, the bioenergetics profile normalised and repeated measures ANOVA were significant for the $\mathrm{Pi} / \mathrm{PCr}$ ratio $(p=0.014)$. We observed an increase in the $\mathrm{Pi} / \mathrm{PCr}$ ratio during visual stimulation and a decrease during recovery using paired $t$ tests $(\mathrm{p}=0.021)$. The increased $\mathrm{Pi} / \mathrm{PCr}$ ratio during brain activation reflected a proportional elevation of ADP, allowing increased mitochondrial ATP production with triheptanoin. After treatment withdrawal, the f-MRS profile returned to its preintervention abnormal state (figure 1B).

\section{DISCUSSION}

Treatment with triheptanoin promptly reduced the number of non-epileptic paroxysmal manifestations in children and adults with GLUT1-DS. This marked clinical response was associated with a significant production of C5-ketone bodies and the normalisation of the f-MRS bioenergetics profile during brain activation. Despite the absence of a control group, the magnitude of the intervention effect ( $90 \%$ reduction) combined with the metabolic responses argues against a placebo effect. The lack of change in effort-based outcome measures tests such as the
6MWT and 9HPT during treatment further argues against a generalised placebo effect in this study.

Our current observation in GLUT1-DS is supported by previous preclinical and clinical studies. In animal models of diseases associated with brain energy deficiency, triheptanoin improved energy metabolism and motor deficits. ${ }^{11-14}$ The first clinical response on neurological deficits with triheptanoin was reported in PC deficiency. ${ }^{6}$ Triheptanoin was also able to correct the bioenergetics profile in the brain of early affected patients with HD. ${ }^{7}$ Recently, a study in epileptic patients with GLUT1-DS showed a reduction in spike-waves on EEG about $90 \mathrm{~min}$ after taking antiepileptic drugs and triheptanoin. ${ }^{15}$ Despite the small sample size, our study provides strong evidence for a sustainable clinical improvement with triheptanoin in GLUT1-DS together with a robust metabolic response using a validated biomarker of brain energy metabolism. ${ }^{7}$ The confirmation of our data in a larger controlled study would hold promise for an alternative therapeutic approach in GLUT1-DS, especially for patients who cannot comply with the constraints of ketogenic diets.

\section{Author affiliations}

${ }^{1}$ Inserm U 1127, CNRS UMR 7225, Sorbonne Universités, UPMC Univ Paris 06 UMR S 1127, Institut du Cerveau et de la Moelle épinière, ICM, Paris, France ${ }^{2}$ Department of Genetics, AP-HP, Pitié-Salpêtrière University Hospital, Paris, France ${ }^{3}$ Department of Neurology, AP-HP, Pitié-Salpêtrière University Hospital, Paris, France ${ }^{4}$ Department of Neuropediatrics, AP-HP, Robert Debré University Hospital, Paris, France

${ }^{5}$ Department of Dietetics, AP-HP, Pitié-Salpêtrière University Hospital, Paris, France

${ }^{6}$ Department of Neuropediatrics, AP-HP, Armand Trousseau University Hospital, Paris, France

${ }^{7}$ Department of Neuropediatrics, Gui de Chauliac Hospital, Montpellier, France ${ }^{8}$ INSERM, U-1051, Institute of Neuroscience, Montpellier, France

${ }^{9}$ Department of Neurology, AP-HM, La Timone University Hospital, Marseille, France

${ }^{10}$ Center for Neurolmaging Research (CENIR), Institut du Cerveau et de la Moelle épinière, Paris, France

${ }^{11}$ Biochemistry and Genetic Laboratory, AP-HP, Bichat-Claude Bernard Hospital, Paris, France

${ }^{12}$ Baylor Research Institute, Institute of Metabolic Disease, Dallas, Texas, USA ${ }^{13}$ Metabolic Biochemistry Lab, AP-HP, Necker University Hospital, Paris, France

${ }^{14}$ University Paris Descartes, Paris, France

${ }^{15}$ Neuromuscular Physiology and Evaluation Lab, Institute of Myology, Paris, France

${ }^{16}$ Service of Clinical Research and Databases, Institute of Myology, Paris, France

Acknowledgements Ultragenyx Pharmaceutical Inc provided the investigational drug triheptanoin and funded the study. The research leading to these results has received funding from the programme 'Investissements d'avenir' ANR-10-IAIHU-06.

The authors are grateful to Dr Isabelle An, Pr Jean-Christophe Corvol and Constance Flamand-Roze for their expertise on some of the material revised for this study, Dr Cécile Hubsch for referral of a patient and Maxime Janin for excellent technical assistance.

Contributors FM was involved in the study concept and design, obtaining funding study supervision and coordination, analysis and interpretation of data, statistical analysis and drafting/revising the manuscript. EH and DG were involved in the study coordination, acquisition of data, analysis and interpretation of data and drafting/ revising the manuscript. IMA was involved in the acquisition of data, statistical analysis of data and drafting/revising the manuscript. SC was involved in the dietary management of the patient and analysis and interpretation of data. BH, AR and EK were involved in the referral of patients and drafting/revising the manuscript. RV, CO and $\mathrm{J}-\mathrm{YH}$ were involved in the acquisition of data and drafting/revising the manuscript. DR and LS were involved in study supervision and coordination and drafting/revising the manuscript. SV was involved in the acquisition of genetic data and drafting/revising the manuscript. RS was involved in the study concept and design and drafting/revising the manuscript. ER was involved in the study concept and design, analysis and interpretation of data, and drafting/revising the manuscript.

Funding ANR 'Investissements d'avenir' (grant no. ANR-10-IAIHU-06) and Ultragenyx Pharmaceutical Inc.

Competing interests FM has a patent application regarding the use of triheptanoin in GLUT1-DS (WO2014093901) and received research support from Ultragenyx and Ipsen; travel funding from Genzyme; and honorarium on an advisory board from Ultragenyx. EH received travel funding from Naturalia and Biologia. $\mathrm{BH}$ received honorarium as board membership from Biomarin, grants and travel and meeting expenses funding from Actelion, travel and meeting expenses funding from 
Shire-HGT. RS has a patent application regarding the use of triheptanoin in GLUT1-DS (W02014093901). RS received research support and honoraria from Genzyme Corporation, Shire and Amicus Therapeutics. JY-H received research funding from Roche and Valerion therapeutics. LS received research funding from Roche and Valerion therapeutics. ER received research funding from Merz-Pharma Orkyn, IP santé and Ultragenyx; served on scientific advisory boards for Orkyn and Merz-pharma; received speech honorarium from Merz-pharma, Novartis, Ipsen-Pharma and Orkyn, received travel funding from Ipsen-Pharma, Teva, Abbvie and Merz-Pharma.

Patient consent Obtained.

Ethics approval Comité de Protection des Personnes, lle-de-France VI (Paris 6).

Provenance and peer review Not commissioned; externally peer reviewed.

Open Access This is an Open Access article distributed in accordance with the Creative Commons Attribution Non Commercial (CC BY-NC 4.0) license, which permits others to distribute, remix, adapt, build upon this work non-commercially, and license their derivative works on different terms, provided the original work is properly cited and the use is non-commercial. See: http://creativecommons.org/ licenses/by-nc/4.0/

\section{REFERENCES}

1 De Vivo DC, Trifiletti RR, Jacobson RI, et al. Defective glucose transport across the blood-brain barrier as a cause of persistent hypoglycorrhachia, seizures, and developmental delay. N Engl J Med 1991;325:703-9.

2 Leen WG, Klepper J, Verbeek MM, et al. Glucose transporter-1 deficiency syndrome: the expanding clinical and genetic spectrum of a treatable disorder. Brain 2010;133:655-70.

3 Gras D, Roze E, Caillet S, et al. GLUT1 deficiency syndrome: an update. Rev Neurol (Paris) 2014;170:91-9.
4 Pons $\mathrm{R}$, Collins $\mathrm{A}$, Rotstein $\mathrm{M}$, et al. The spectrum of movement disorders in Glut-1 deficiency. Mov Disord 2010;25:275-81.

5 Klepper J, Leiendecker B. Glut1 deficiency syndrome and novel ketogenic diets. J Child Neurol 2013;28:1045-8.

6 Mochel F, DeLonlay P, Touati G, et al. Pyruvate carboxylase deficiency: clinical and biochemical response to anaplerotic diet therapy. Mol Genet Metab 2005;84:305-12.

7 Adanyeguh IM, Rinaldi D, Henry PG, et al. Triheptanoin improves brain energy metabolism in patients with Huntington disease. Neurology 2015;84:490-5.

8 Sappey-Marinier D, Calabrese G, Fein G, et al. Effect of photic stimulation on human visual cortex lactate and phosphates using $1 \mathrm{H}$ and 31P magnetic resonance spectroscopy. J Cereb Blood Flow Metab 1992;12:584-92.

9 Mochel F, N'Guyen TM, Deelchand D, et al. Abnormal response to cortical activation in early stages of Huntington disease. Mov Disord 2012;27:907-10.

10 Chance B, Eleff $\mathrm{S}$, Leigh JS, et al. Mitochondrial regulation of phosphocreatine/ inorganic phosphate ratios in exercising human muscle: a gated 31P NMR study. Proc Natl Acad Sci USA 1981;78:6714-18.

11 Kim TH, Borges K, Petrou S, et al. Triheptanoin reduces seizure susceptibility in a syndrome-specific mouse model of generalized epilepsy. Epilepsy Res 2013;103:101-5

12 Marin-Valencia I, Good LB, Ma Q, et al. Heptanoate as a neural fuel: energetic and neurotransmitter precursors in normal and glucose transporter I-deficient (G1D) brain. J Cereb Blood Flow Metab 2013;33:175-82.

13 Hadera MG, Smeland OB, McDonald TS, et al. Triheptanoin partially restores levels of tricarboxylic acid cycle intermediates in the mouse pilocarpine model of epilepsy. J Neurochem 2014;129:107-19.

14 Francis JS, Markov V, Leone P. Dietary triheptanoin rescues oligodendrocyte loss, dysmyelination and motor function in the nur7 mouse model of Canavan disease. J Inherit Metab Dis 2014;37:369-81.

15 Pascual JM, Liu P, Mao D, et al. Triheptanoin for glucose transporter type I deficiency (G1D): modulation of human ictogenesis, cerebral metabolic rate, and cognitive indices by a food supplement. JAMA Neurol 2014;71:1255-65. 